\title{
WIP: Using neuro-responses to understand creativity, the engineering design process, and concept generation
}

\section{Tess Hartog, University of Oklahoma}

Tess Hartog is a graduate student in Mechanical Engineering at the University of Oklahoma. Her interests include creativity, engineering education, and neuroimaging. Her research focuses on understanding creativity and divergent thinking in engineering students via the use of electroencephalography (EEG).

\section{Megan Marshall, The University of Oklahoma}

Megan Marshall is an M.S. Aerospace Engineering candidate at the School of Aerospace and Mechanical Engineering, The University of Oklahoma. Her research interests include the neuroscience of creativity and design, and using these insights to develop a person's creative and design ability.

\section{Mr. Amin G. Alhashim, U of Oklahoma}

Amin G. Alhashim is a Ph.D. candidate at the School of Industrial and Systems Engineering, The University of Oklahoma. Amin is studying creativity in the field of engineering education and looking forward to leveraging machine learning to deliver more personalized learning for engineers to foster their creativity.

\section{Md Tanvir Ahad, School of Aerospace and Mechanical Engineering, The University of Oklahoma}

Md Tanvir Ahad received his Bachelor's degree from Ahsanullah University of Science and Technology on November2013 in Electrical and Electronic Engineering. From January 2015July 2015 he was at the Dana Engineering International Ltd (GEWaukesha) in Dhaka, Bangladesh as an Assistant Engineer (GEWaukesha) gas engine. From 2015-2018 he was at the Applied DSP Laboratory of the Lamar University at Beaumont,TX, USA, and obtained a Master's degree in Electrical Engineering on 2018. Currently he is perusing his Ph.D. degree in Aerospace and Mechanical Engineering at The University of Oklahoma starting from 2018present. Md Tanvir Ahad currently holds a Graduate research assistant position at Product and Process Design Lab under the advisement of Professor Dr. Zahed Siddique.

\section{Prof. Zahed Siddique, University of Oklahoma}

Zahed Siddique is a Professor of Mechanical Engineering at the School of Aerospace and Mechanical Engineering of University of Oklahoma. His research interest include product family design, advanced material and engineering education. He is interested in motivation of engineering students, peer-to-peer learning, flat learning environments, technology assisted engineering education and experiential learning. $\mathrm{He}$ is the coordinator of the industry sponsored capstone from at his school and is the advisor of OU's FSAE team. 


\title{
WIP: Using neuro-responses to understand creativity, the engineering design process, and concept generation
}

\begin{abstract}
Investigations of creativity have been an intriguing topic for a long time, but assessing creativity is extremely complex. Creativity is a cornerstone of engineering disciplines, so understanding creativity and how to enhance creative abilities through engineering education has received substantial attention. Fields outside of engineering are no stranger to neuro-investigations of creativity and although some neuro-response studies have been conducted to understand creativity in engineering, these studies need to map the engineering design and concept generation processes better. Using neuroimaging techniques alongside engineering design and concept generation processes is necessary for understanding how to improve creative idea generation and creativity studies in engineering. In this paper, a survey is provided of the literature for the different neurological approaches that have been used to study the engineering design process and creative processes. Also presented are proposed strategies to apply these neurological approaches to engineering design to understand the creative process in greater detail. Furthermore, results from a pilot study investigating neuro-responses of engineers are presented.
\end{abstract}

\section{Introduction}

Intelligence, measured by IQ and SAT, has been in a steady increase in America since 1990 [1, 2]. On the other hand, creativity, measured by Torrance Tests of Creative Thinking (TTCT), a widely used and validated measure [3-5] proposed by Ellis Paul Torrance in 1966, has been in steady decline since then $[1,2]$. The creative ability is the most or among the most important, core, and necessary skills to national prosperity in the 21 st century [6, 7]. Hence, nurturing it and/or slowing its decline is vital. Many researchers argued for immediate and serious actions at different levels, starting from parents and ending with nations $[1,2]$.

The National Academy of Engineering has noted that there is a need for creative, as well as competent, engineers $[8,9]$. The desire for creativity in engineers has been noted since around the 1960s [10-12] and has continued to be a desirable aspect $[5,13]$. However, students graduating from engineering fields are lacking the creative ability [14-16] even though creativity and innovation are assumed to be hallmarks of engineering [17, 18]. Moreover, creativity is considered a necessary prerequisite to innovation [15], which means any decline in the creative ability will lead to a decline in the ability of engineers to be innovative.

A survey by [16] at the University of Connecticut found that there is a lack of creativity in the engineering curriculum that is taught, and that students believed educators focused on the use of conventional solutions to problems rather than novel solutions. At the same time, though, instructors claimed to value creativity but didn't see it in their students. Similarly, a study by [19] reported that as students move forward in their engineering education, they believe that creativity is not highly valued. Furthermore, many researchers have found that the engineering discipline 
has become more focused on convergent thinking and rote learning as opposed to other, more innovative approaches $[9,20-28]$.

Fortunately, research has shown that creative ability is like a muscle and can be trained and enhanced via certain types of processes, exercises, and techniques. Studies performed by [29] and [30] showed through both behavioral and neuroscientific methods that the creative ability can be trained and enhanced by showing that the brain activates differently after using creativity enhancing exercises and techniques. Though using behavioral approaches to study the impact of these processes, exercises, and techniques on creativity is useful, the use of these approaches does not provide a direct way to investigate the causes that underlie creativity, which may lead to contradicting conclusions. Neurological approaches can provide a direct way to study these underlying processes.

The use of neuroimaging allows researchers to have visible, physical results that connect creativity to biological processes and structures. These techniques give us a better, more accurate view of creativity via the direct acquisition of objective, quantitative data versus the indirect transformation of qualitative behavioral data into quantifiable data. This allows researchers to have a more direct, clear-cut view on whether methods claiming to improve creativity actually do so. Methods that are said to aid in innovative design could be utilized with neuroimaging techniques to gain quantifiable measurements directly related to these methods. Instead of relying on human-based scoring methods alone (which are subjective), the use of neuroimaging could give us a better objective understanding as to how creative an individual truly is.

In this paper, we investigate the development of new experimental approaches that can relate widely used engineering design and concept generation techniques with neuroimaging techniques. First, we provide information about two main neuroimaging techniques and how they work (Section 2) and discuss the engineering design process (Section 3). Then, we provide an overview of how neuroimaging has been used in conjunction with engineering design in the past with the two selected techniques (Section 4). Additionally, we present results of a pilot study conducted by our lab investigating creativity via the use of EEG (Section 5). Finally, we suggest future research into the application of neuroimaging techniques to the engineering design process in order to further understand and improve creativity in engineering and design education (Section 6).

\section{Neuroimaging Methods}

There are many neuroimaging techniques that have been used to investigate creativity: PET [31], SPECT [32], NIRS [33, 34], and DTI [35, 36] are just a few that have been used. In this paper, we focus on two of the most commonly used ones: functional magnetic resonance imaging (fMRI) and electroencephalography (EEG). These methods are introduced below, along with a discussion of their strengths and weaknesses. For more comprehensive reviews of these techniques, see [37-41]. 


\subsubsection{Functional Magnetic Resonance Imaging (fMRI)}

The most used neuroscience technique to investigate creativity is the functional magnetic resonance imaging (fMRI) technique [41]. The fMRI technique works by applying a strong magnetic field to measure the changes in the ratio of oxygenated to deoxygenated blood in the brain. As brain activity occurs, blood is transported to the active parts of the brain to deliver oxygen to sustain brain processes [40]. Measuring this change in ratio allows brain activity to be physically mapped with a high spatial resolution. Unfortunately, as delivery of oxygenated blood is an after effect of brain activity meant to replenish and sustain processes, temporal resolution is low, with a built-in time lag. Though the low temporal resolution is a drawback of this method, its high spatial resolution capabilities have made it a popular choice for studies focusing on what physical areas of the brain are most active during specific processes. Another advantage of this method is that it is noninvasive, which makes it a cleaner, simpler process compared to those that utilize the injection of radioactive tracers into subjects [40]. Though this method is noninvasive, it does require the patient to lay in an fMRI machine with as little movement as possible. This limits the types and duration of tasks that can be studied as well as the responses a subject can give to a task. fMRI trials tend to only last about forty minutes, including trial blocks of stimulation tasks, response times, and pauses. Compared to other methods, though, fMRI does allow for longer trial periods, which allows researchers to obtain more statistically significant data. Working in a magnetic environment though, does add the disadvantage of limiting types of data collection hardware to those with non-magnetic components [40]. These limitations of data collection hardware and communication to researchers of subjects' responses leads to responses being communicated after trial blocks. Though this overcomes the problem of interference during the fMRI, answers may be completely forgotten, changed, or elaborated upon after the trial block.

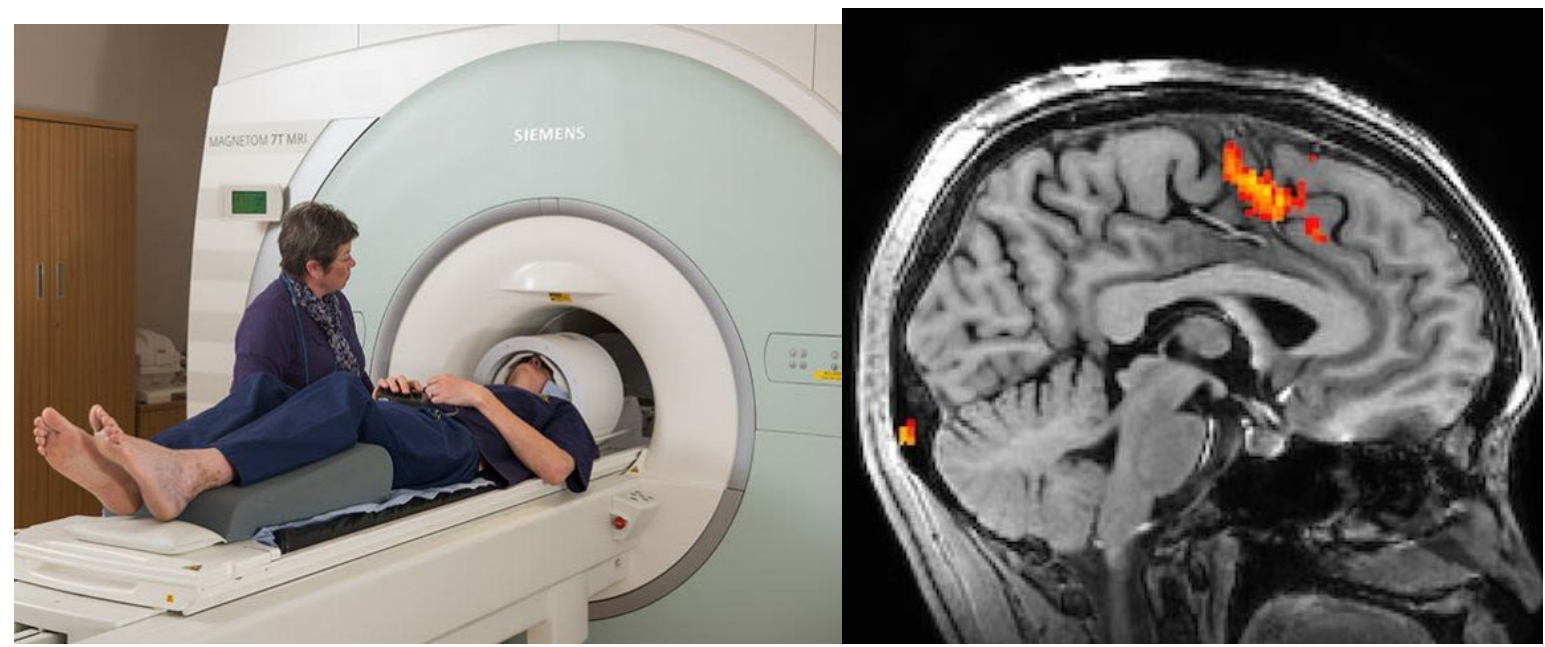

Figure 1 - MRI machine and image produced from BOLD contrast taken from [42].

\subsubsection{Electroencephalogram (EEG)}

Another technique used in creativity research is the electroencephalogram (EEG). An EEG is a device used to measure and record the "...electrical potentials generated in the extracellular fluid as ions flow across cell membranes and neurons talk to one another via neurotransmitters" [43]. 
These electrical signals are collected through electrodes placed on the scalp. From these signals, responses to stimuli can be extracted and analyzed, providing high temporal resolution of brain activity. EEG signals are analyzed based on frequency, amplitude, and electrode position.

Frequency bands such as delta $(0.1-4 \mathrm{~Hz})$, theta $(4-8 \mathrm{~Hz})$, alpha $(8-13 \mathrm{~Hz})$, beta $(13-30 \mathrm{~Hz})$, and gamma $(30-100 \mathrm{~Hz})$ relate to specific states of brain activity, and these states can be mapped to various areas of the brain with high temporal accuracy.
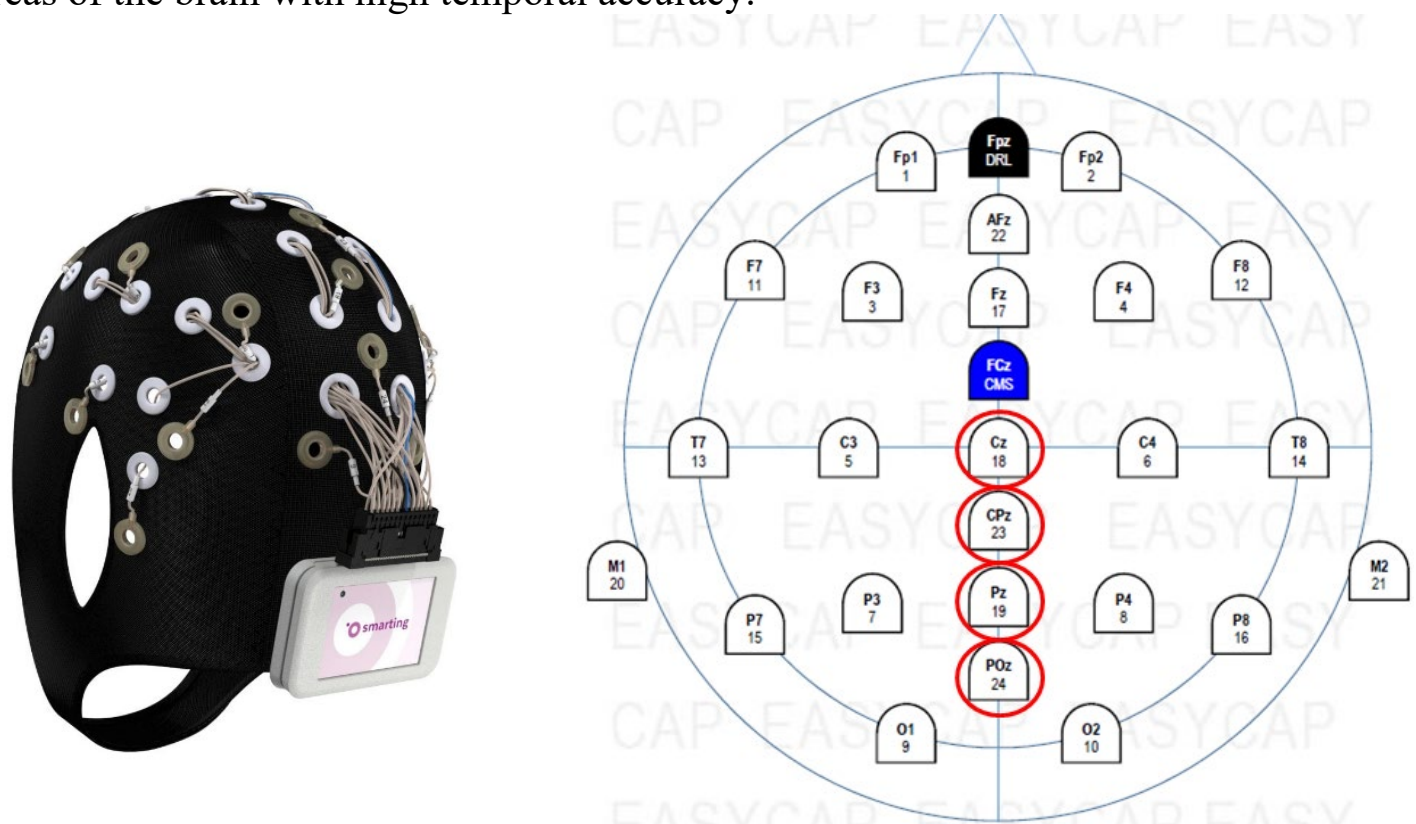

Figure 2 - Mobile EEG cap with 24 channels and corresponding electrode layout taken from [44]. Electrodes circled (Cz, CPz, Pz, and POz) are for reference later in the text (Section 5.2).

Alpha waves have been noted in various studies to correlate to tasks requiring creative responses. Many of these studies have examined a phenomenon called alpha synchronization, a period when alpha frequency (activity around the alpha band of $8-13 \mathrm{~Hz}$ ) increases in power. The synchronization period is associated with periods of cognitive idling or rest. Alpha desynchronization, on the other hand, is related to a loss of power in the alpha frequency band and typically presents when cognition is actively engaged. Increased alpha synchronization had been linked to greater creative ability [45, 46] as well as more original ideas [47-49]. Several researchers have also reported that creative training was related to higher alpha activity, thus indicating the possibility that creative ability can be enhanced [50-52].

EEGs can also be used to record event-related potentials (ERPs). ERPs are signals that are timelocked to a stimulus and provide a step by step visualization of the brain processes at each electrode during a trial [40]. They are direct measurements, down to the millisecond, of neurotransmitter activity [53]. Several components, noted as positive or negative signal amplitude peaks or fluctuations correlated to specific times, have been discovered that relate to specific brain processes. Specifically, the N400, post-N400, and P50 components have been related to creative processes. The N400 is a negatively (signified by the "N") peaking potential that occurs between $300-500 \mathrm{~ms}$ after stimulus presentation. It has been related to the processing of semantic mismatches and violations of prior knowledge [40]. Additionally, a study by [54] linked the N400 component to conceptual expansion and noticed it responds to unusual stimuli. 
Similarly, [55] reported the N400 as responsive as a function of unusualness or novelty to their experimental stimuli while investigating conceptual expansion. The post-N400 component is a negative response that varies directly following the N400 component and is related to interpretation processes and concept integration. Similarly, the P50 is a positively (signified by the "P") peaking potential occurring around $50 \mathrm{~ms}$ after stimulus presentation. This component is related to sensory gating of relevant and irrelevant information.

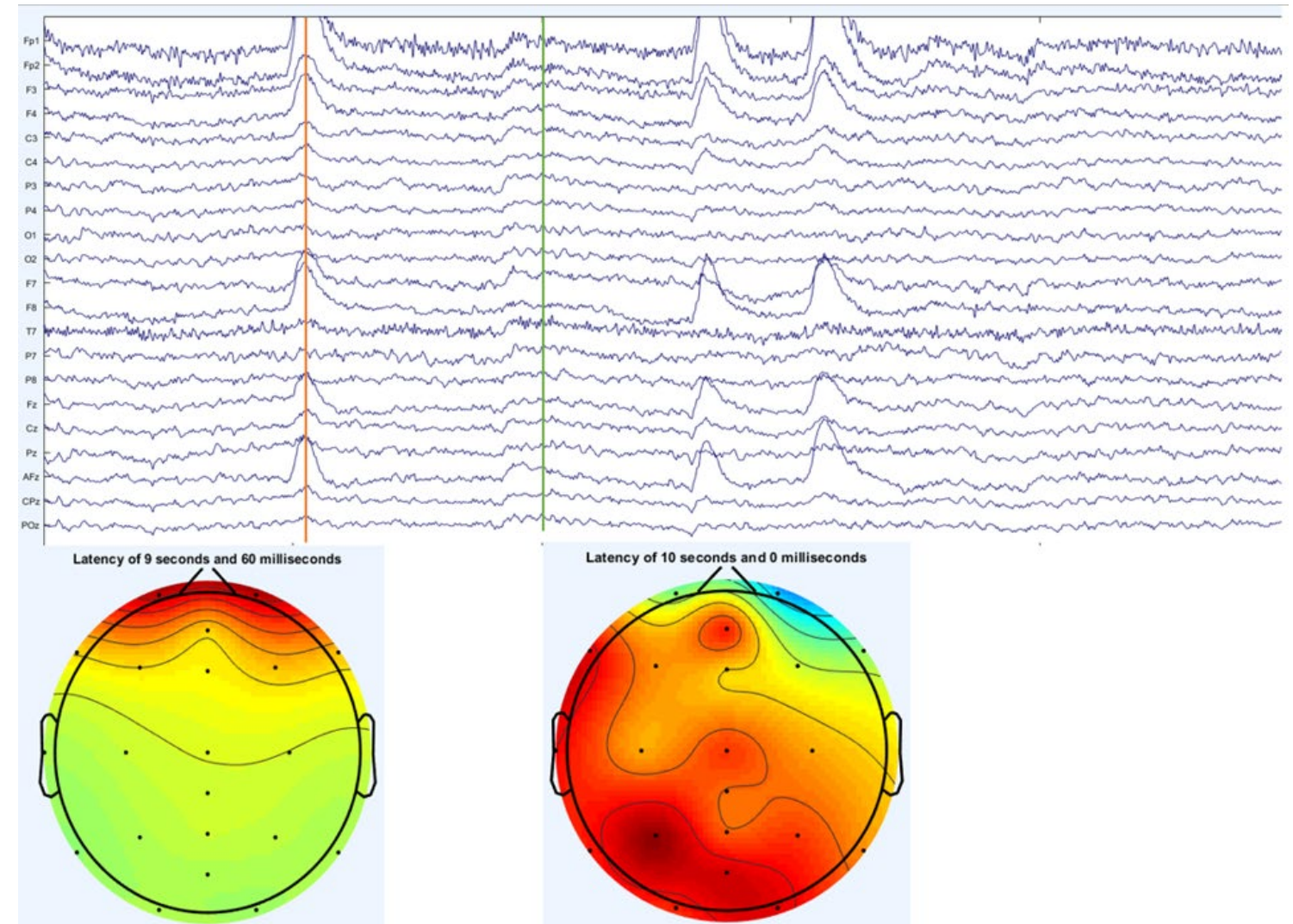

Figure 3 - Raw EEG data from 24 electrodes filtered between .5-100 Hz (Top) and corresponding electrical activity head maps at two points (bottom).

Because of its high temporal precision, the use of EEG and ERP in studies are ideal for providing data about the neural processes that occur between stimulus presentation and neural response. Currently, ERP has been used to understand language processing and Alternative Usage Task experiments. Overall, measuring temporal variation of neuro-response during idea generation can provide ways to better understand creative thinking by allowing us to measure creative ideas and relate them with neuro-responses. 


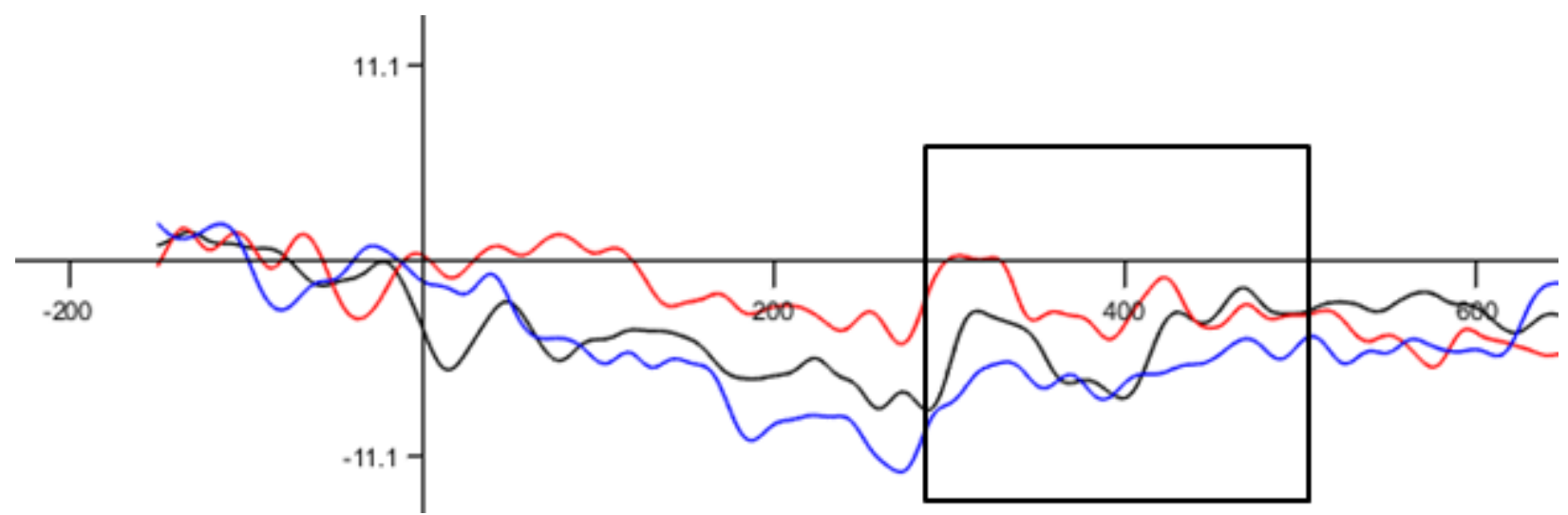

Figure 4 - ERP images from one electrode for three different types of stimuli. Box represents the $300 \mathrm{~ms}-500 \mathrm{~ms}$ post-stimulus range, where the N400 effect could be found.

\section{The Engineering Design Process}

In this section is presented a generalized engineering design process to which neuroscientific techniques could be applied to understand creativity and divergent thinking in engineering design and concept generation (ED\&CG). As discussed in Section 1, it is imperative to develop creativity in engineers. Creativity is key to providing innovative solutions to unique and difficult problems. One process that engineers use to solve these problems is the engineering design process. Though there are several different groups of thought and various specific methods and techniques, the basic process is as follows:

1. Identify the problem or need

2. Generate possible solutions

3. Downselect one or more solutions

4. Prototype the solution

5. Test and evaluate the solution

Each step is its own iterative process, and if necessary, steps may be repeated to obtain a final solution. In the first step, a problem or need is identified and researched. Stakeholders, requirements, and constraints all need to be established to understand the actual problem to be addressed. Once the "actual" problem has been established, step two can commence, and solutions can be generated that address this problem. In step 3, generated solutions are downselected to a small number to prototype, allowing for important tradeoffs to be made and money to be saved from prototyping less appropriate solutions. Once a prototype reaches step 5, if it fails or needs improvement, engineers may go back to step 3 or even step 2 . If it succeeds, it may be further improved or exit the design process and continue to manufacturing. See Figure 5. 


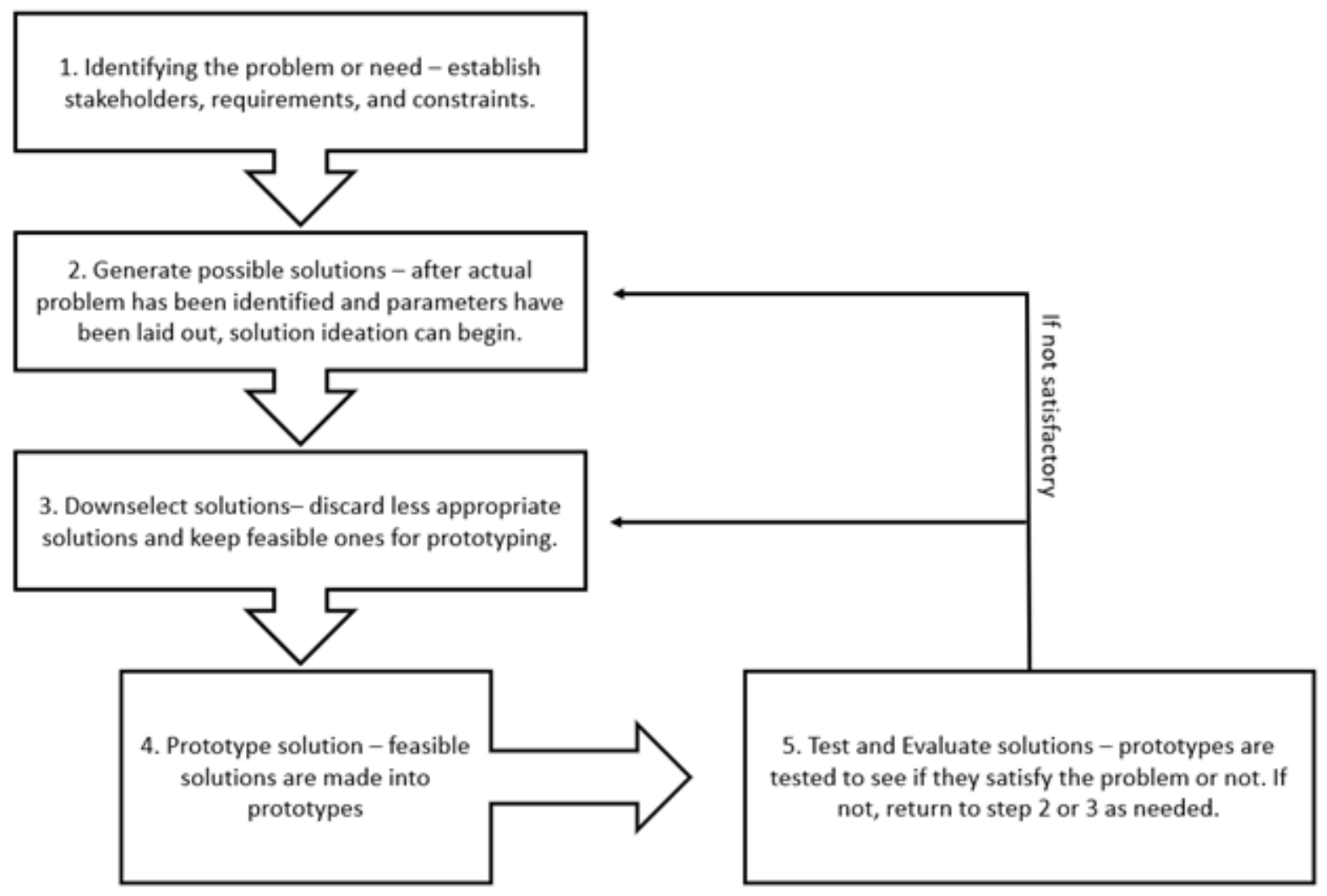

Figure 5 - Typical Engineering Design Process

Creativity in this process is important, but how does one measure the amount of creativity that an engineer uses during this process? This kind of process is not executed in a vacuum. Engineers must work in teams, communicate with stakeholders, integrate new knowledge, and use various methods, techniques, and tools. Each process takes days and weeks to complete. How can a complicated process like this be broken down and studied from a neuroscientific point of view? Table 1 shows what needs to be studied in relation to the engineering design process. Use of techniques for spatial investigations (such as fMRI, etc.) can shed light on which areas of the brain are active during certain parts of the engineering design process. Next, since spatial investigations find out which areas of the brain are active, the corresponding areas can be investigated using temporal methods (i.e., EEG). Overall, the location alone is not enough for understanding how to change engineering curriculum. There is a specific need to focus on temporal investigations with respect to the generation of solutions to problems. The use of timebased techniques will advance research since these experiments will show exactly when idea generation occurs (down to the millisecond), what prompted the idea or what was happening when it occurred, and how that prompt or action can be used in education. This will be especially useful for creative or novel idea generation. 
Table 1 - Neuroimaging investigations needed for the engineering design process.

\begin{tabular}{|l|c|c|}
\hline \multicolumn{1}{|c|}{ Design Process Step } & $\begin{array}{c}\text { Spatial (Location) } \\
\text { Investigation }\end{array}$ & $\begin{array}{c}\text { Temporal (Time) } \\
\text { Investigation }\end{array}$ \\
\hline $\begin{array}{l}\text { 1 - Identifying the problem or } \\
\text { need }\end{array}$ & $\checkmark$ & $\checkmark \checkmark$ \\
\hline 2- Generate possible solutions & $\checkmark$ & \\
\hline $\begin{array}{l}\text { 3 - Downselect one or more } \\
\text { solutions }\end{array}$ & $\checkmark$ & \\
\hline 4 - Prototype the solution & $\checkmark$ & \\
\hline $\begin{array}{l}\text { 5- Test and evaluate the } \\
\text { solution }\end{array}$ & $\checkmark$ & \\
\hline
\end{tabular}

\section{Utilizing Neurological Techniques to Study Engineering Design}

Though neuroimaging methods have been used to investigate general concepts and theories of creativity, application of these methods to investigate creativity in engineering design and concept generation has been minimal. An important aspect to consider when selecting a neuroimaging method is whether the device has better spatial resolution or temporal resolution. Spatial resolution relates to a method's capability to provide fine location detail, to map the areas of the brain experiencing activity. Temporal resolution refers to the granularity of time detail obtained when brain activation is occurring. Different techniques offer different combinations of spatial and temporal resolution, but typically fMRI is regarded as having high spatial resolution and poor temporal resolution, while EEG is the opposite, having poor spatial resolution and excellent temporal resolution. Thus, technique type needs to be taken into consideration when designing experiments based upon what is to be examined. In this section, some of the current neurological investigations of engineering design and concept generation (ED\&CG) are presented. Overall, there is a limited amount of work that has been published relating neuroimaging and design [56]. There are some studies that make use of fMRI and EEG, but at this time, no papers were found applying ERP to engineering design type problems.

\subsection{Current use of fMRI in the study of ED\&CG}

One of the first design investigations was conducted by [57] who utilized fMRI to study the difference in the cognitive processes employed when solving design tasks compared to nondesign tasks. The authors found that design tasks and non-design tasks employ different cognitive processes. These cognitive processes are linked to different regions of the brain and there was extensive activation of these regions when solving the design tasks compared to the non-design tasks. The paper further suggests that general problem-solving and design thinking are distinct. Although [57] is not a plain study of creativity, the methodology used to study the design process can be adopted when studying creativity in the ED\&CG process. 
A study by [58] used fMRI to investigate design ideation and concept generation with and without the support of inspirational stimuli (e.g., analogies). While not all participants were in the field of engineering, participants were graduate-level students specializing in engineering, design, or product development, which included mechanical engineering students. Results indicated that brain activation was different for participants that were able to successfully use the inspiration to generate an insightful design and those that were unsuccessful (mostly those that did not receive inspiration).

The study in [59] used fMRI to investigate brain activity of engineering designers during conceptual generation in order to see if design fixation (defined as adherence to a set of ideas or concepts that limit the final output of a design) could be detected when participants were solving design problems. Some participants were given example images (sketches) and others were not. Areas of the brain associated with creative output were found to be less active in the example condition.

\subsection{Current use of EEG in the study of ED\&CG}

Researchers from Concordia University have done a case study analyzing design activities via EEG [60]. In this research, the participant was given a design problem that asked the participant to arrange furniture in a room based on a given set of circumstances and measurements. In a follow-up study by [61], engineering students were asked to design a house that could fly while EEG was recorded. This experiment used a technique called clustering that examined the power spectral density in the different halves of the brain, but there were no significant results. Yet another study by [62] recorded EEG and heart rate while engineering students worked on a design problem of their choice, however most picked the same house design problem as listed before. They found that mental effort (which they used as an indirect measure of creativity and measured via EEG) was lowest when mental stress is highest (measured via the heart rate monitor). These small-scale studies indicate that it is possible to use EEG alongside design-type problems, yet it is complex due to a multitude of factors such as the intricacies involved in the engineering design process, the associated processing of data, and the isolation of creative thought processes.

A recent EEG study by [63] replicated the [57] study mentioned above (Section 4.1). The experiment consisted of 18 mechanical engineering students and 18 architects. The experimental setup incorporated an additional open design task that included free-hand sketching. Findings indicated design neurocognition differed when problem-solving versus designing, particularly in the sketching task, as indicated by transformed power and task-related power within the EEG readings.

\subsection{Current use of other techniques in the study of ED\&CG}

While not a primary talking point of this paper, a notable investigation with the use of functional near infrared spectroscopy (fNIRS) is worthy of mention. This technique works via the absorption or reflection of hemoglobin in certain areas of the brain. Researchers in [64] utilized fNIRS to investigate the neurological differences in freshman and senior-level engineering students during an engineering design brainstorm. Even though this study did not look at the 
novelty of ideas generated, this study found that freshmen generated more solutions and had five times greater activation in regions of the brain related to memory, planning, decision making, and ability to think about multiple concepts at once than seniors. On the other hand, seniors had ten times the activation in areas associated with behavior control, uncertainty management, and self-reflection in decision making.

\section{Preliminary ERP investigation}

As noted above, there are currently no ERP based experiments of engineering design and creativity. Furthermore, as of date, our research has not found any ERP studies related to engineering in any aspect. In order to investigate ERPs near the realm of engineering, our lab has run a pilot study investigating the N400 response of engineers following a design similar to the experiment in [55]. Two male individuals, one in Aerospace and Mechanical Engineering and the other in Industrial and Systems Engineering, participated in one trial each for this pilot study. Their results were averaged together for further analysis and then presented here. A brief overview of the experiment will follow. While this experiment was not a direct investigation of engineering design, this experiment presents promising results related to engineering and neuroresponses. Additionally, since the basis of investigations of this type are not present, it is necessary to complete studies of this type in order to construct a base to build upon. Once the basics are covered, there are many different possibilities for neurological research in engineering, which are discussed in the next section of this paper (Section 6).

\subsection{The Study}

The pilot study presented here is based off the study that Kroger et. al. [55] implemented in an experimental effort to look at ERPs as an investigation of conceptual expansion. Their team investigated cognitive expansion as a central component of creative thinking based off of a 2012 study [54], which found that conceptual expansion was linked to the N400 component. Kroger et al. [55] looked at ERP data from 24 students from their university with unspecified majors in order to relate the N400 component to unusualness or novelty of stimuli via the use of a modified alternative usage task (AUT). Traditionally, the AUT asks participants to generate as many alternative uses as possible for a common object, such as a pencil. Instead of generating uses for a given item, though, participants were shown a word of an object in conjunction with a potential use for that object as a stimulus. There were three categories of stimuli: creative uses (i.e., Shoe $>$ Pot Plant), nonsense uses (i.e., Shoe > Easter Bunny), and common uses (i.e., Shoe > Clothing). Participants were then asked to decide if the given use was unusual and if it was appropriate and they would answer these questions by pushing buttons. Our pilot study narrows down the general focus of [55] to investigate results of individuals solely from engineering.

\subsection{Results}

Results from this study follow a similar pattern of [55], which indicates that the N400 component is sensitive to semantic difference as well as novelty which is indicated by differences in the mean amplitudes of the four electrodes of interest for each stimulus type, see Figure 6 . The four electrodes of interest $(\mathrm{Cz}, \mathrm{CPz}, \mathrm{Pz}$, and $\mathrm{POz}$ ) were chosen here based upon electrodes identified in [54]. Locations of these electrodes are highlighted in Figure 2. Data indicated that stimuli 
classified as nonsensical or creative elicit larger N400 amplitudes than the common uses. Given the higher amplitudes for the nonsense and creative uses, it is an indication that the N400 in engineers is sensitive to levels of novelty or unusualness.

In summary, data from this pilot study indicates that the $\mathrm{N} 400$ component in engineers is influenced by novelty and unusualness. In the future, we aim to increase the number of participants in order to validate the pilot study, investigate the post-N400 response, and remove the POz electrode from analysis due to its ties to vision as opposed to creative thinking processes. The waveforms of single electrode sites $\mathrm{Cz}$ and $\mathrm{CPz}$ from one of the trials are depicted below in Figure 7.

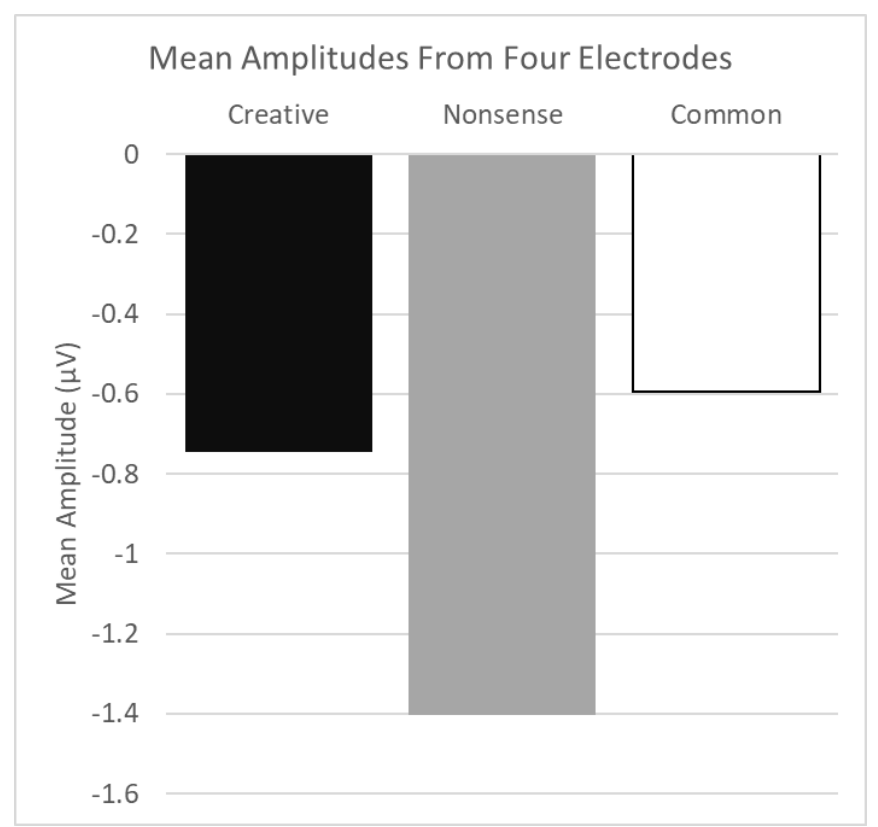

Figure 6 - Mean amplitudes from four electrodes $(\mathrm{Cz}, \mathrm{CPz}, \mathrm{Pz}$, and $\mathrm{POz})$ for the three types of item-use pairs (creative uses, nonsensical uses, and common uses) for the $300-500 \mathrm{~ms}$ time window investigating the $\mathrm{N} 400$ effect. 

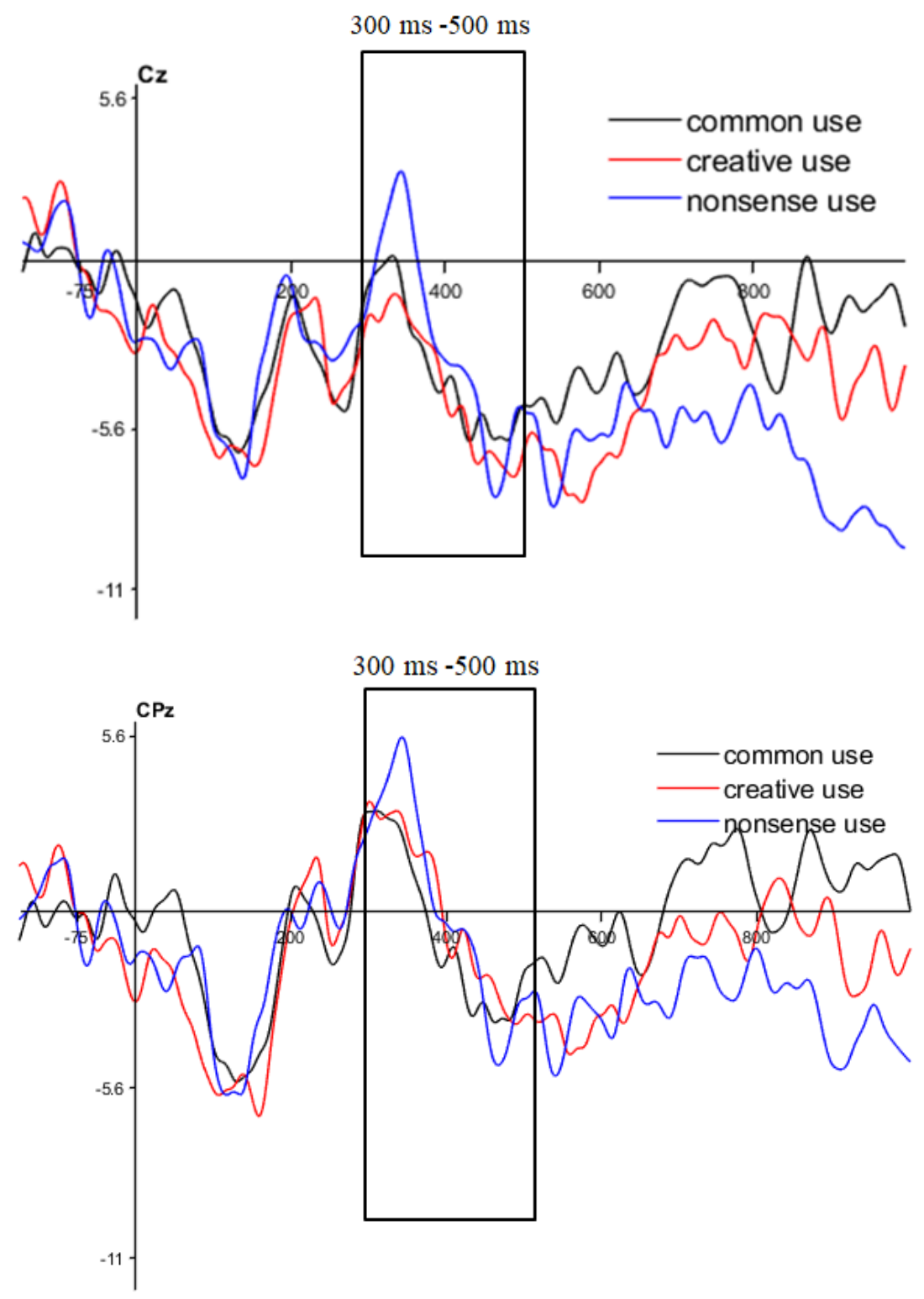

Figure 7 - ERPs from the $\mathrm{Cz}$ (top) and $\mathrm{CPz}$ (bottom) electrodes from one individual. The box outline indicates the 300-500 ms window of investigation of the N400 effect.

\section{Future Directions}

Designing experiments to study the neurological responses on engineering design and concept generation is not a straightforward task. However, by using tools like fMRI, areas of the brain that are active during the engineering design process could be highlighted. This would be achieved by designing experiments such that fMRI could be recorded while addressing different parts of the engineering design process or during idea generating tasks. Then, since the general area of interest would be known, this would allow further investigations with other methods like EEG, and appropriate approaches to EEG experiments could be determined. In this paper, we focus more on potential EEG experiments than on fMRI, and present two experimental concepts for future investigations. 
One important consideration when studying engineering design and concept generation is breaking up these complex, multi-step processes into neuroscientifically measurable processes. The studies reported in Section 4 treated the problem solving and design process as a black box (i.e., as a single, long step). However, when wanting to investigate the effect of a treatment on a single part of the problem solving or design process (e.g., the idea generation step or the problem finding step), there is work needed to design these experiments.

For example, no research has yet been completed on studying the effect of using the alternative uses task (AUT) on engineering students' divergent thinking skills when generating concepts to solve an engineering problem. This could be accomplished as follows. Two groups of engineering students would be asked to solve a design problem. One group would be asked to practice with the AUT before solving the engineering problem, and the other group (the control group) would solve the engineering problem without practicing with the AUT. The AUT portion of the experiment would not have to be monitored with an EEG, as the creativity will be measured from the engineering problem. The engineering problem would be broken into two steps. First, participants would be presented with a problem that requires a solution (e.g., prevent water from sticking to a glass surface). Then, participants would be asked to generate simple alternative ideas that would satisfy the problem. After the idea generation step, the participants would be asked to use their concepts to come up with one complete solution. EEG would be recorded for the entirety of the engineering portion of the experiment. These signals could then be compared to find differences between the two groups. It would also be possible to utilize ERP, as well. In this case, the participant could press a button every time they think of a solution and the ERP could be analyzed around that time.

Since no ERP studies related to engineering design and concept generation have been done, including how to improve instructions for these approaches, there is a need for further investigations into this area. In designing ERP experiments, it is important to identify components of interest (i.e., N400). As mentioned in Section 2.1.2, the N400 or Post-N400 components would be a good place to start since studies have shown there is some relation to novelty, unusualness, and conceptual expansion [54, 55]. Since ERPs are responses to stimuli, it is important to have ED\&CG experiments broken up into small, manageable parts, as suggested above. Methods like function structure diagrams and Energy-Material-Systems (EMS) models are useful in breaking down engineering problems into smaller chunks and thus could be used to design short and simple experiments appropriate for ERP analysis. For instance, it would be possible to present certain aspects of a problem to a participant followed by possible solutions (e.g., selecting an energy source for a machine followed by different types of energy sources such as solar power, wind power, natural gas, etc.) to measure the effect a suggested solution has on brain activity. On the same note, it would be possible to present a problem to a participant and have them press a button when they generate their own possible solution all while recording EEG (e.g., telling participant to come up with possible energy sources for a machine and pressing a button when they come up with a solution).

With neurological research into engineering design and concept generation starting to bud, there are several sub-areas that could be further investigated. Potential experiments include studying creativity and improving instruction at different stages of the engineering design process (as suggested above), studying the effects of different models and techniques such as EMS, TRIZ, 
etc. on ideation, studying creative responses and idea generation within teams, studying the effects of diversity within teams on the engineering design process, and studying the effect of experience on creative responses and idea generation.

Through these experiments, various processes, exercises, and techniques used to improve an individual's creativity could be tested for their effectiveness. The neuro-responses during concept generation and steps of the engineering design process could also be used to understand how the brain operates during these activities. Specific EEG frequencies and ERP components could be identified as key to concept generation and specific steps in the design process. These responses could then be related to experimental data from specific creativity improving processes, exercises, and techniques to obtain targeted improvement of specific brain processes. These processes, exercises, and techniques could then be appropriately implemented within the engineering curriculum to effectively improve students' creativity. The neuro-responses can inform which design tools to use at different steps of the design process and how to improve instructions on proper application of the tools.

\section{Conclusion}

Since creativity is not well understood with respect to engineering [15, 21, 65], nor is there a lot of previous neuroscientific research investigating ED\&CG, it is difficult to design experiments related to the topic. In this paper, we discussed neuroimaging techniques as well as how these techniques have been used in relation to ED\&CG up to this point. We also provided suggestions for experiments, and the next step would be to conduct these investigations. As more data from these future investigations becomes available, it can be used to improve engineering education. This data will aid researchers in understanding what cognitive processes are used in the engineering design process. Furthermore, creativity improving techniques could be measured using neuroscientific data. These techniques could then be incorporated into engineering education curriculum to promote creativity in engineers.

\section{Acknowledgement}

This material is based upon work supported by the National Science Foundation under Grant No. 1561660 and 1726358, 1726811, and 1726884. Any opinions, findings, and conclusions or recommendations expressed in this material are those of the author(s) and do not necessarily reflect the views of the National Science Foundation. 


\section{References}

[1] K. H. Kim, "The Creativity Crisis: The Decrease in Creative Thinking Scores on the Torrance Tests of Creative Thinking," Creativity Research Journal, vol. 23, no. 4, pp. 285-295, 2011.

[2] K. H. Kim and R. A. Pierce, "Torrance's innovator meter and the decline of creativity in America," The Routledge International Handbook of Innovation Education, vol. 5, pp. 153-167, 2013.

[3] G. A. Davis, "Testing for creative potential," Contemporary Educational Psychology, vol. 14, no. 3, pp. 257-274, 1989.

[4] K. H. Kim, "Can We Trust Creativity Tests? A Review of the Torrance Tests of Creative Thinking (TTCT)," Creativity Research Journal, vol. 18, no. 1, pp. 3-14, Jan. 2006.

[5] H. B. Parkhurst, "Confusion, Lack of Consensus, and the Definition of Creativity as a Construct," The Journal of Creative Behavior, vol. 33, no. 1, pp. 1-21, 1999.

[6] R. Florida, The rise of the creative class--revisited: Revised and expanded. Basic Books (AZ), 2014.

[7] "IBM 2010 Global CEO Study: Creativity Selected as Most Crucial Factor for Future Success," IBM News room - 2010-05-18 IBM 2010 Global CEO Study: Creativity Selected as Most Crucial Factor for Future Success - United States, 18-May-2010. [Online]. Available: https://www-03.ibm.com/press/us/en/pressrelease/31670.wss. [Accessed: 12-Mar-2020].

[8] National Academy of Engineering, U. S. The engineer of 2020: Visions of engineering in the new century. Washington, DC: National Academies Press, 2004.

[9] Olson, Steve, ed. Educating engineers: Preparing 21st century leaders in the context of new modes of learning: Summary of a forum. National Academies Press, 2013.

[10] F. E. Jones, "Predictor variables for creativity in industrial science.," Journal of Applied Psychology, vol. 48, no. 2, pp. 134-136, 1964.

[11] C. D. Mcdermid, "Some correlates of creativity in engineering personnel.," Journal of Applied Psychology, vol. 49, no. 1, pp. 14-19, 1965.

[12] T. B. Sprecher, "A study of engineers criteria for creativity.," Journal of Applied Psychology, vol. 43, no. 2, pp. 141-148, 1959.

[13] B. D. Bleedorn, "Creativity: Number One Leadership Talent for Global Futures," The Journal of Creative Behavior, vol. 20, no. 4, pp. 276-282, 1986.

[14] K. Bateman, "IT students miss out on roles due to lack of creativity," ComputerWeekly, 18Apr-1013. [Online]. Available: https://www.computerweekly.com/blog/ITWorks/ITstudents-miss-out-on-roles-due-to-lack-of-creativity. [Accessed: 12-Mar-2020].

[15] D. H. Cropley, "Creativity in engineering." In Multidisciplinary contributions to the science of creative thinking, pp. 155-173. Springer, Singapore, 2016.

[16] K. Kazerounian and S. Foley, "Barriers to Creativity in Engineering Education: A Study of Instructors and Students Perceptions," Journal of Mechanical Design, vol. 129, no. 7, pp. 761-768, 2007.

[17] C. Charyton, Creative engineering design assessment: background, directions, manual, scoring guide and uses. London: Springer, 2014.

[18] L. Richards, "Stimulating creativity: teaching engineers to be innovators," FIE 98. 28th Annual Frontiers in Education Conference. Moving from Teacher-Centered to LearnerCentered Education. Conference Proceedings (Cat. No.98CH36214), vol. 3, pp. 10341039, Nov. 1998. 
[19] C. Masters, S. Hunter, and G. Okudan. "Design Process Learning and Creative Processing: Is There a Synergy." In ASEE Conference Proceedings. 2009.

[20] J. P. Adams, S. Kaczmarczyk, P. Picton, and P. Demian. "Improving problem solving and encouraging creativity in engineering undergraduates." From International Conference on Engineering Education, 2007.

[21] D. H. Cropley, "Promoting creativity and innovation in engineering education.," Psychology of Aesthetics, Creativity, and the Arts, vol. 9, no. 2, pp. 161-171, 2015.

[22] J. J. Duderstadt, "Engineering for a Changing Road, a Roadmap to the Future of Engineering Practice, Research, and Education.," 2007

[23] C.L. Dym, A. M. Agogino, O. Eris, D. D. Frey, and L. J. Leifer. "Engineering design thinking, teaching, and learning." Journal of engineering education 94, no. 1, pp. 103 120, 2005.

[24] R. M. Felder, "Creativity in engineering education." Chemical Engineering Education 22, no. 3, pp. 120-125, 1988.

[25] J. A. Plucker, R. A. Beghetto, and G. T. Dow, "Why Isnt Creativity More Important to Educational Psychologists? Potentials, Pitfalls, and Future Directions in Creativity Research," Educational Psychologist, vol. 39, no. 2, pp. 83-96, 2004.

[26] J. C. Santamarina, "Creativity and Engineering-Education Strategies." Proc. Int. Conference on Engineering Education in Honor of JTP Yao, Texas A\&M, pp. 91-108, 2003.

[27] R. Stratton, D. Mann, and P. Otterson. "The Theory of Inventive Problem Solving (TRIZ) and Systematic Innovation-a Missing Link in Engineering Education?." TRIZ Journal, 2000.

[28] S. Törnkvist, "Creativity: Can It Be Taught? The Case of Engineering Education," European Journal of Engineering Education, vol. 23, no. 1, pp. 5-12, 1998.

[29] E. Gregory, M. Hardiman, J. Yarmolinskaya, L. Rinne, and C. Limb, "Building creative thinking in the classroom: From research to practice," International Journal of Educational Research, vol. 62, pp. 43-50, 2013.

[30] C. J. Limb and A. R. Braun, "Neural Substrates of Spontaneous Musical Performance: An fMRI Study of Jazz Improvisation," PLoS ONE, vol. 3, no. 2, 2008.

[31] N. Bechtereva, A. Korotkov, S. Pakhomov, M. Roudas, M. Starchenko, and S. Medvedev, "PET study of brain maintenance of verbal creative activity," International Journal of Psychophysiology, vol. 53, no. 1, pp. 11-20, 2004.

[32] R. A. Chávez-Eakle, A. Graff-Guerrero, J.-C. García-Reyna, V. Vaugier, and C. CruzFuentes, "Cerebral blood flow associated with creative performance: A comparative study," NeuroImage, vol. 38, no. 3, pp. 519-528, 2007.

[33] B. S. Folley and S. Park, "Verbal creativity and schizotypal personality in relation to prefrontal hemispheric laterality: A behavioral and near-infrared optical imaging study," Schizophrenia Research, vol. 80, no. 2-3, pp. 271-282, 2005.

[34] C. Gibson, B. S. Folley, and S. Park, "Enhanced divergent thinking and creativity in musicians: A behavioral and near-infrared spectroscopy study," Brain and Cognition, vol. 69, no. 1, pp. 162-169, 2009.

[35] H. Takeuchi, Y. Taki, Y. Sassa, H. Hashizume, A. Sekiguchi, A. Fukushima, and R. Kawashima, "Regional gray matter volume of dopaminergic system associate with creativity: Evidence from voxel-based morphometry," NeuroImage, vol. 51, no. 2, pp. 578-585, 2010. 
[36] H. Takeuchi, Y. Taki, Y. Sassa, H. Hashizume, A. Sekiguchi, A. Fukushima, and R. Kawashima, "White matter structures associated with creativity: Evidence from diffusion tensor imaging," NeuroImage, vol. 51, no. 1, pp. 11-18, 2010.

[37] L. M. Pidgeon, M. Grealy, A. H. B. Duffy, L. Hay, C. Mcteague, T. Vuletic, D. Coyle, and S. J. Gilbert, "Functional neuroimaging of visual creativity: a systematic review and meta-analysis," Brain and Behavior, vol. 6, no. 10, Nov. 2016.

[38] A. Fink and M. Benedek, "EEG alpha power and creative ideation," Neuroscience \& Biobehavioral Reviews, vol. 44, pp. 111-123, 2014.

[39] R. Arden, R. S. Chavez, R. Grazioplene, and R. E. Jung, "Neuroimaging creativity: A psychometric view," Behavioural Brain Research, vol. 214, no. 2, pp. 143-156, 2010.

[40] A. Abraham, The neuroscience of creativity. Cambridge, United Kingdom: Cambridge University Press, 2019.

[41] A. Dietrich and R. Kanso, "A review of EEG, ERP, and neuroimaging studies of creativity and insight.," Psychological Bulletin, vol. 136, no. 5, pp. 822-848, 2010.

[42] Nuffield Department of Clinical Neurosciences. University of Oxford. Retrieved April 6, 2020, from https://www.ndcn.ox.ac.uk/divisions/fmrib/what-is-fmri/how-is-fmri-used

[43] G. F. Woodman, "A brief introduction to the use of event-related potentials in studies of perception and attention," Attention, Perception, \& Psychophysics, vol. 72, no. 8, pp. 2031-2046, 2010.

[44] MBrainTrain. Fully Mobile EEG Devices. Retrieved February 3, 2020, from https://mbraintrain.com/, 2018.

[45] A. Fink, R. H. Grabner, M. Benedek, G. Reishofer, V. Hauswirth, M. Fally, C. Neuper, F. Ebner, and A. C. Neubauer, "The creative brain: Investigation of brain activity during creative problem solving by means of EEG and FMRI," Human Brain Mapping, vol. 30, no. 3, pp. 734-748, 2009.

[46] E. Jauk, M. Benedek, and A. C. Neubauer, "Tackling creativity at its roots: Evidence for different patterns of EEG alpha activity related to convergent and divergent modes of task processing," International Journal of Psychophysiology, vol. 84, no. 2, pp. 219-225, 2012.

[47] A. Fink and A. C. Neubauer, "EEG alpha oscillations during the performance of verbal creativity tasks: Differential effects of sex and verbal intelligence," International Journal of Psychophysiology, vol. 62, no. 1, pp. 46-53, 2006.

[48] R. H. Grabner, A. Fink, and A. C. Neubauer, "Brain correlates of self-rated originality of ideas: Evidence from event-related power and phase-locking changes in the EEG.," Behavioral Neuroscience, vol. 121, no. 1, pp. 224-230, 2007.

[49] D. Schwab, M. Benedek, I. Papousek, E. M. Weiss, and A. Fink, "The time-course of EEG alpha power changes in creative ideation," Frontiers in Human Neuroscience, vol. 8, 2014.

[50] A. Fink, R. H. Grabner, M. Benedek, and A. C. Neubauer, "Divergent thinking training is related to frontal electroencephalogram alpha synchronization," European Journal of Neuroscience, vol. 23, no. 8, pp. 2241-2246, 2006.

[51] A. Fink, R. H. Grabner, M. Benedek, and A. C. Neubauer, "Divergent thinking training is related to frontal electroencephalogram alpha synchronization," European Journal of Neuroscience, vol. 23, no. 8, pp. 2241-2246, 2006. 
[52] A. Fink, D. Schwab, and I. Papousek, "Sensitivity of EEG upper alpha activity to cognitive and affective creativity interventions," International Journal of Psychophysiology, vol. 82, no. 3, pp. 233-239, 2011.

[53] S. Luck, An Introduction to the Event-Related Potential Technique. Second edition. Cambridge, Massachusetts: The MIT Press, 2014.

[54] B. Rutter, S. Kröger, H. Hill, S. Windmann, C. Hermann, and A. Abraham, "Can clouds dance? Part 2: An ERP investigation of passive conceptual expansion," Brain and Cognition, vol. 80, no. 3, pp. 301-310, 2012.

[55] S. Kröger, B. Rutter, H. Hill, S. Windmann, C. Hermann, and A. Abraham, "An ERP study of passive creative conceptual expansion using a modified alternate uses task," Brain Research, vol. 1527, pp. 189-198, 2013.

[56] T. Shealy, and M. Hu. "Evaluating the potential of neuroimaging methods to study engineering cognition and project-level decision making." EPOC-MW Conference, Engineering Project Organization Society, Fallen Leaf Lake, CA USA, 2017.

[57] K. Alexiou, T. Zamenopoulos, J. Johnson, and S. Gilbert, "Exploring the neurological basis of design cognition using brain imaging: some preliminary results," Design Studies, vol. 30, no. 6, pp. 623-647, 2009.

[58] K. Goucher-Lambert, J. Moss, and J. Cagan, “A neuroimaging investigation of design ideation with and without inspirational stimuli-understanding the meaning of near and far stimuli," Design Studies, vol. 60, pp. 1-38, 2019.

[59] K. K. Fu, B. Sylcott, and K. Das, "Using fMRI to deepen our understanding of design fixation," Design Science, vol. 5, 2019.

[60] T. A. Nguyen, T. An, and Y. Zeng. "Analysis of design activities using EEG signals." ASME 2010 international design engineering technical conferences and computers and information in engineering conference. American Society of Mechanical Engineers Digital Collection, 2010.

[61] T. A. Nguyen, and Y. Zeng. "Clustering designers' mental activities based on EEG power." Tools and methods of competitive engineering, Karlsruhe, Germany, 2012.

[62] T. A. Nguyen and Y. Zeng, "A physiological study of relationship between designer's mental effort and mental stress during conceptual design," Computer-Aided Design, vol. 54, pp. 3-18, 2014.

[63] S. L. D. S. Vieira, J. S. Gero, J. Delmoral, V. Gattol, C. Fernandes, and A. A. Fernandes, "Comparing the Design Neurocognition of Mechanical Engineers and Architects: A Study of the Effect of Designer's Domain," Proceedings of the Design Society: International Conference on Engineering Design, vol. 1, no. 1, pp. 1853-1862, 2019.

[64] T. Shealy, J. Grohs, M. Hu, D. Maczka, and R. Pannenton, "Investigating design cognition during brainstorming tasks with freshmen and senior engineering students using functional near infrared spectroscopy." ASEE, Columbus, OH, 2017.

[65] R. Jonczyk, J. van Hell, G. Okudan Kremer, and Z. Siddique. "Neurocognitive Evidence on the Impact of Topical Familiarity in Creative Outcomes.", 2019. 